طريقة برمجة الأهداف الخطية لاتخاذ القرار الامثل لمشاكل التخصيص متعدد المعايير

\author{
واثق حياوي لايذ الذفاجي \\ قسم الإحصاء، كلية الإدارة والاقتصاد، جامعة سومر \\ 2019 نشر في: 30 حزير
}

الخلاصة ـ تعد عملية اتخاذ القرارات جو هر العملية الإدارية ووسيلتها في تحقيق أهداف المنظمات الخدمية او الانتاجية وذللك لأن القرار

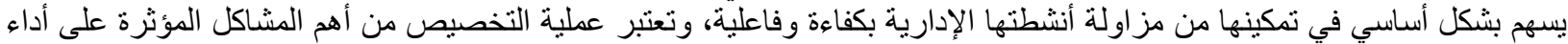

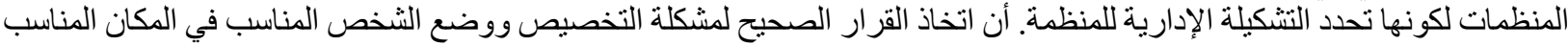

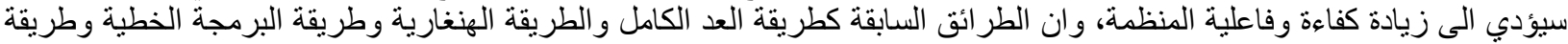

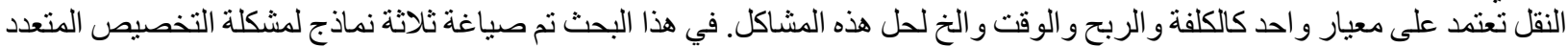

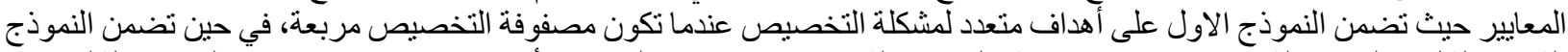

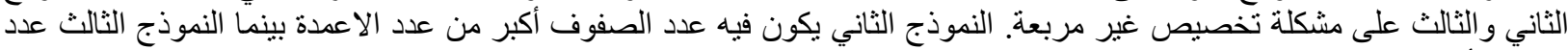

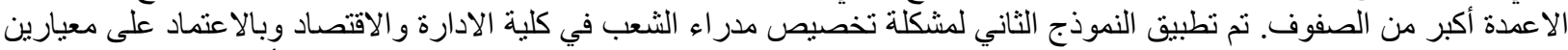

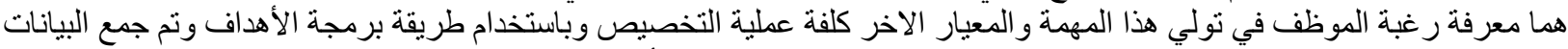

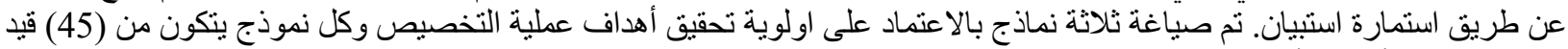

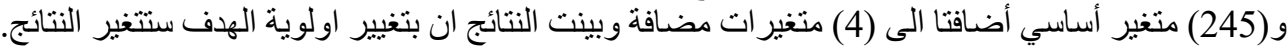

الكلمات الرئيسية -ـ.

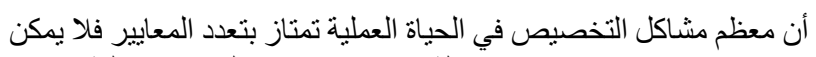

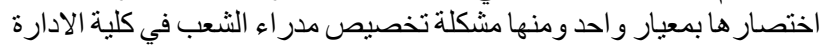

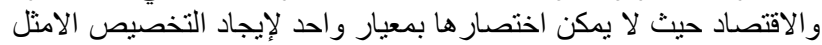

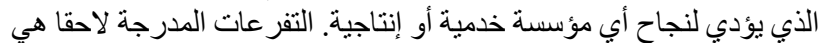

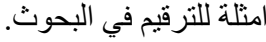

\section{3. - هدف البحث}

أن هدف البحث هو حل مشاكل التخصيص المتعدد المعايير عندما تكون مصفوفة التخصيص مربعة أو غير مربعة وباستخدام طريقة بر مجة الأهداف وتطبيق ذللك لتخصبص مدر اء الشعب في كلية الادارة

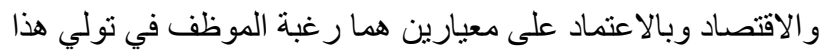

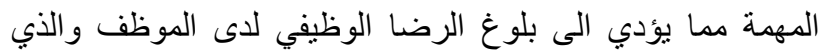

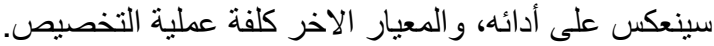

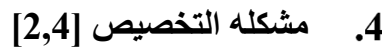

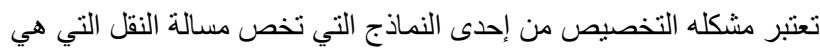

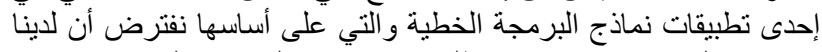

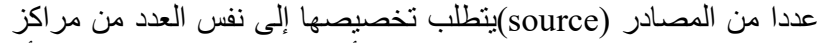

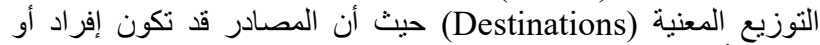

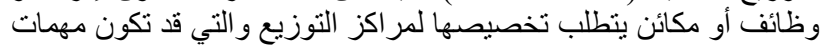
أو إعمال أو مخازن بما يضمن تقليل الوقت لون أو الكلف او تعظيع الأرباح

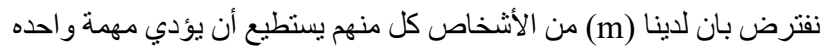

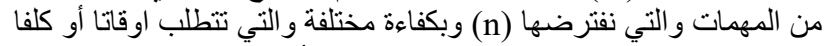

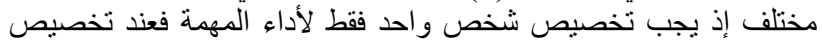

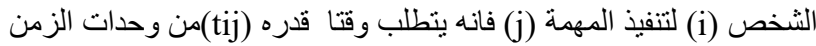

1

إن جوهر الممارسة الإدارية هو اتخاذ القرارات لأجل حل المشاكل اليومية

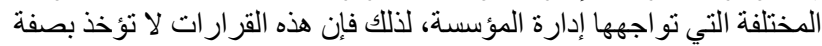

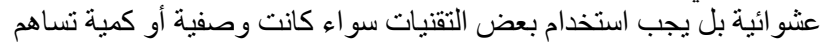

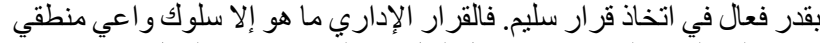

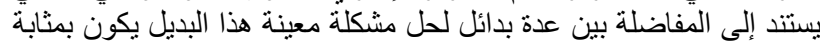

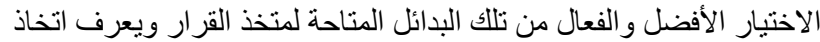

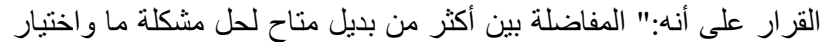

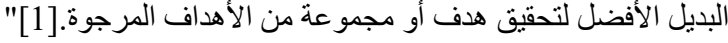

أن استخدام الأسلوب الكمي للتوصل إلى صنع قرارات أكثر دقة وأصالة

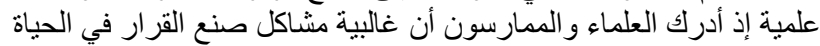

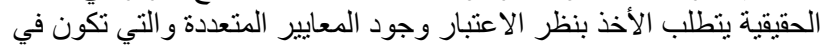

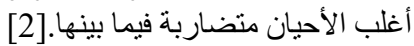

إن من أهم المشاكل التي تواجهها إدارة المنظمات هي كيفية اتخاذ القرار التهار

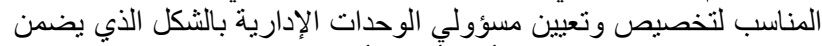

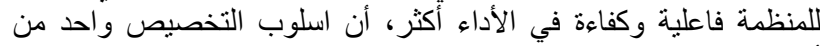

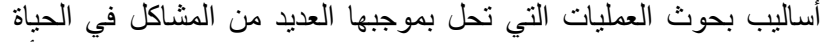

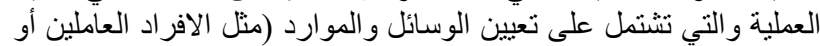

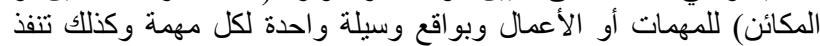

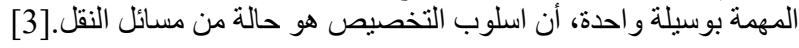

مشكلة البحث

.2

أن الطر ائق السابقة لحل مشكلة التخصيص تعتمد على معيار واحد

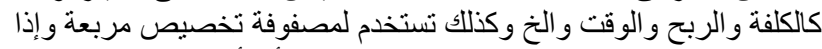

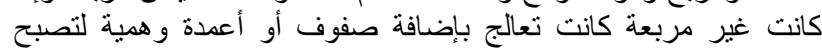
المصفوفة مربعة. 
الثكل (1): مر احل اتخاذ القرارات [6]

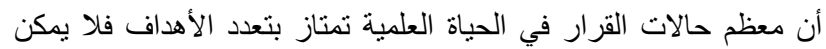

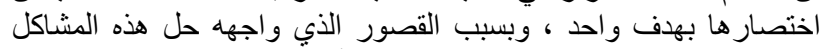

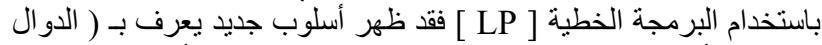

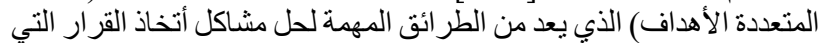

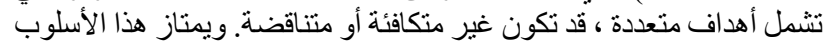

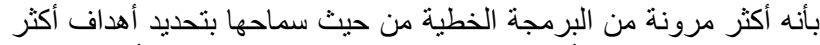

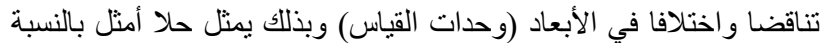

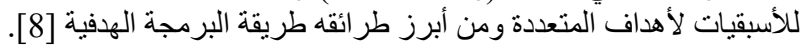

\section{6}

إن الفكرة الأساسية في برمجة الأهداف هي تحديد أولوية لكل هدف ، ثم تحديد

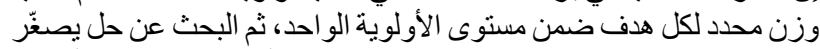

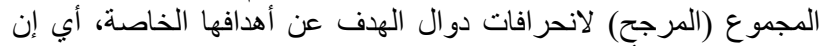

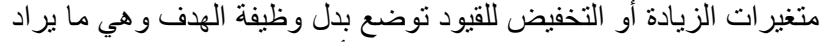

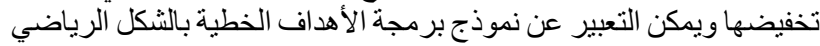

الآتي [5,9]:

Min a - -

$=\{\mathrm{p} 1[\mathrm{~g} 1(\mathrm{~d} 1+, \mathrm{d} 1-)], \mathrm{p} 2[\mathrm{~g} 2(\mathrm{~d} 2+, \mathrm{d} 2-)]$

$, \ldots \ldots, \operatorname{pk}[\mathrm{gk}(\mathrm{di}+, \mathrm{di}-)]\}$

Subject to:

$\sum \mathrm{m}_{\mathrm{i}=1} \mathrm{C}_{\text {in }} \mathrm{X}_{\mathrm{n}}+\mathrm{d}_{\mathrm{i}}{ }^{+}+\mathrm{d}_{\mathrm{i}}^{-}=\mathrm{b}_{\mathrm{i}}$

$\mathrm{i}=1,2,3, \ldots \ldots, \mathrm{m}$

$\mathrm{Xj}, \mathrm{d} i+, \mathrm{d} i-\geq 0$

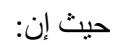

a

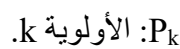

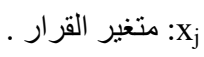

.

لمتغير الانحراف السالب ويعبر وجوده في دالة الإنجاز عن أدنى إنجاز للهذف .

لمتغير الانحر اف الموجب ويعبر وجوده في دالة الإنجاز عن أعلى إنجاز d $^{+}{ }^{+}$ للهذف .

. ق قيمة الهدف bi

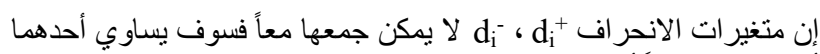

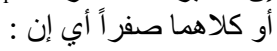

$\mathrm{d}_{\mathrm{i}}^{+} * \mathrm{~d}_{\mathrm{i}}^{-}=0$

كما ينطبق شرط عدم السلبية على جميع المتغيرات ، أي إن :

$d_{i}^{-}, d_{i}^{+} \geq 0$

هنالك ثلاث حالات يمكن إن نقوم بهـا لتقليص متغير ات الانحر اف في دالة

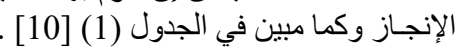

أو كلفه قدر ها (cij) من وحدات العملة لغرض انجاز المهمة ومن ثم تحقيق

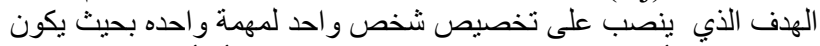

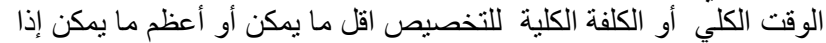

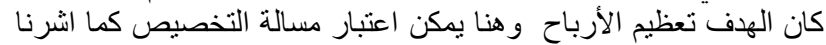

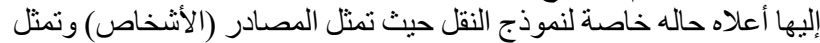
مر اكز التوزيع (المهماتهاتهاتهات

هنالك عدة طر ائق لحل مشاكل التخصيص منها: 1

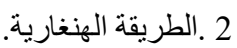

3

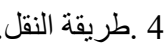

جميع هذه الطر ائق أعلاه تكون عاجزة عن حل مشاكل التخصيص المتعدد

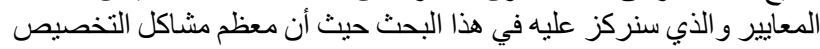

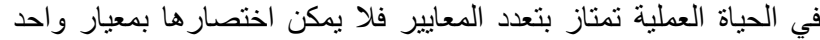

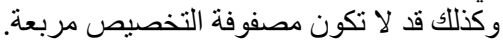

5. اتخاذ القرارات المتعدد المعايير

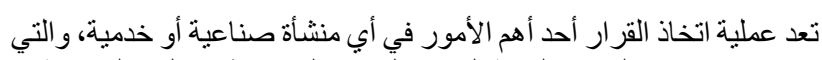

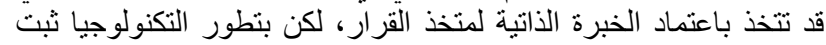

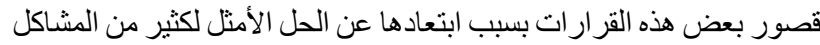

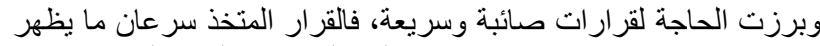

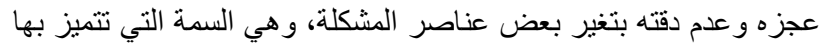

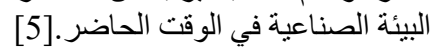

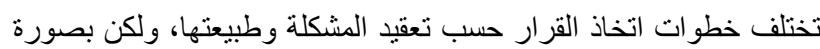

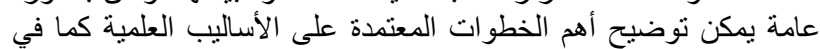

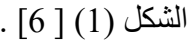

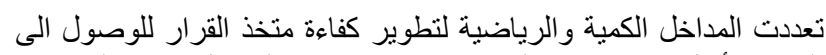

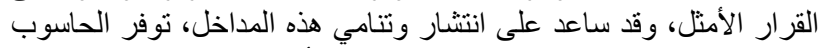

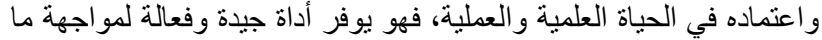

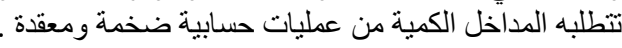

أن أساليب بحوث العمليات (operations research) تستخدم بشكل واسع التع التئ

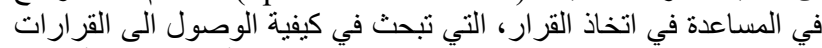

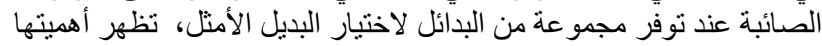

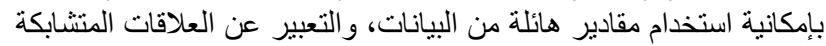

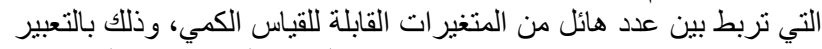

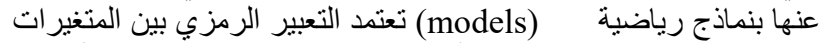

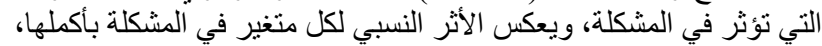

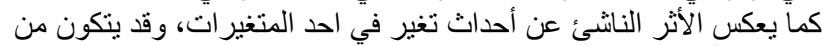

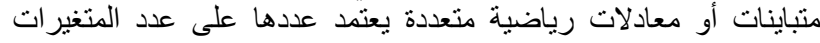

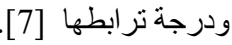

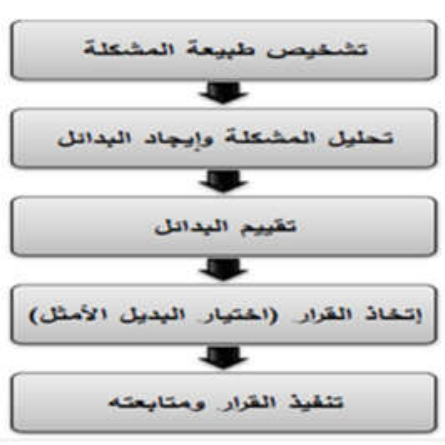




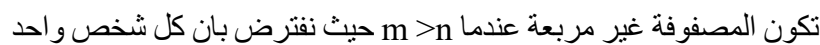

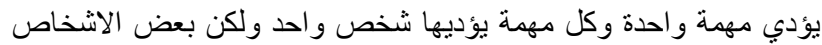

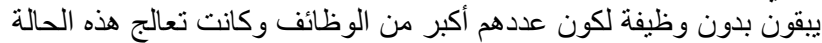

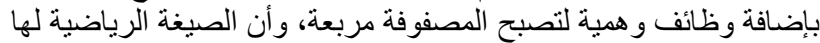

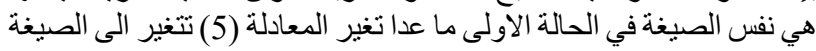

$\sum_{i=1}^{n} X_{i j} \leq 1 \quad$ for all $j=1,2, \ldots, n$

هذه الحالة سوف تطبق في الحالة التطبيقية لبحثنا.

$$
\text { لحالة الثانية (m>n) }
$$

تكون المصفوفة غير مربعة عندما n >m حيث نفترض بان كل شخص و واحد

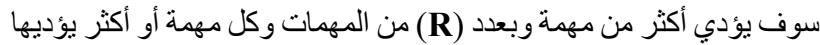

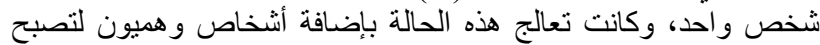

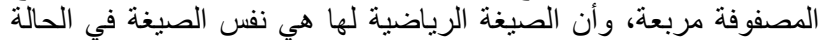

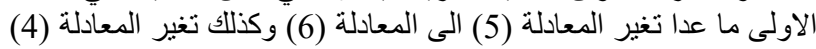

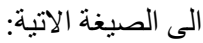

$\sum_{j=1}^{n} X_{i j}=R \quad$ for all $i=1,2, \ldots, m$

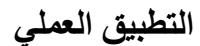

لغرض تطبيق الجزء النظري وتخصيص مدراء الثعب في كلية الادارة

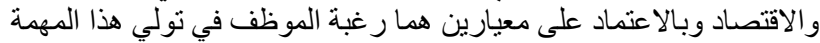

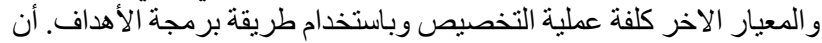

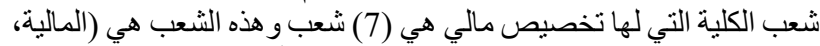

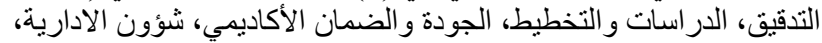
شؤون الطلبة، البحث و التطوير ).

$$
\text { جمع البيانات }
$$

8.1

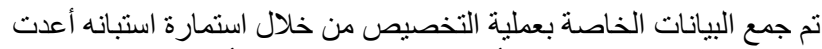

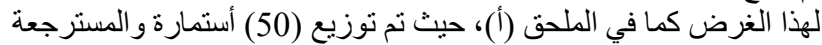
(40) أستمارة وان (35) أستمارة بياناتها كاملة .

تم التعويض عن (لا أتفق بشدة، لا أتفق، محايد، أتفق، أتفق بشدة) بالأرقام

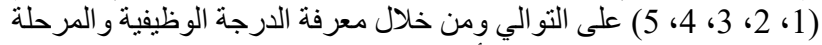

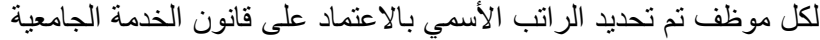

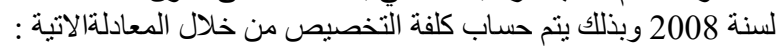

كلفة

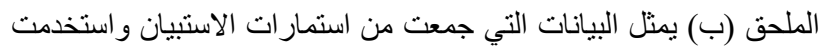

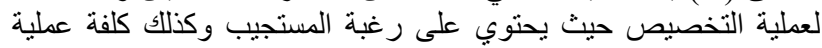

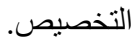

\section{الصياغة الرياضية لمشكلة التخصيص}

8.2

للوصول إلى صياغة رياضية لمشكلة تخصيص مدراء الشعب في كلية

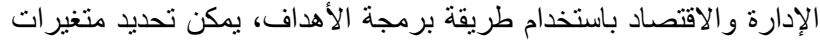
وأهداف وقيود المشكلة وكما يأتي:

8.2.1

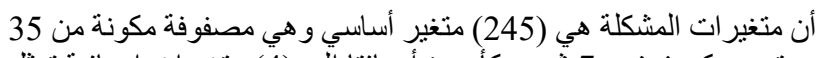

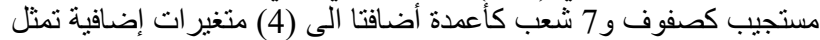

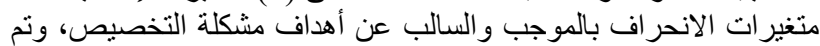

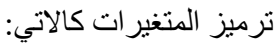

الجدول (1): يبين حالات تخفيض متغيرات الانحراف في دالة الإنجاز

\begin{tabular}{|c|c|c|}
\hline نوع القيد & الصيغة العامة للقيد & الانتراف \\
\hline & $f_{i}(\bar{x})+d_{i}^{-}-d_{i}^{+}=b_{i}$ & $\mathrm{~d}_{\mathrm{i}}^{+}$ \\
\hline$f_{i}(\bar{x}) \geq b_{i}$ & $f_{i}(\bar{x})+d_{i}^{-}-d_{i}^{+}=b_{i}$ & $\overline{\mathrm{d}_{\mathrm{i}}^{-}}$ \\
\hline$f_{i}(\bar{x})=b_{i}$ & $f_{i}(\bar{x})+d_{i}^{-}-d_{i}^{+}=b_{i}$ & $\mathrm{~d}_{\mathrm{i}}^{-}+\mathrm{d}_{\mathrm{i}}^{+}$ \\
\hline
\end{tabular}

لطريق برمجة الاهداف [10] لعالات

الصيغة الرياضية للتخصيص المتعدد المعايير باستخدام .7 طريقة برمجة الأهداف

يمكن تقسيم مشكلة التخصيص الى ثثلاث حالات بالاعتماد على عداد المداد

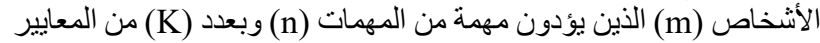
المستخدمة لعملية التخصيص، وكما موضنح في الحالات أدناه

الحالة الأولى (m=n)

7.1

تكون المصفوفة مربعة عندما m=n وهي الحالة المطلوبة لأي عملية

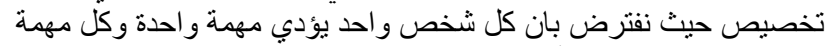

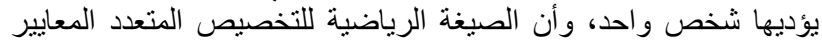
باستخدام طريقة برمجة الأهداف هو كالاتي:

$\operatorname{MinZ}_{1}\left(\operatorname{or} M a x Z_{1}\right)=\sum_{i=1}^{m} \sum_{j=1}^{n} t_{i j} X_{i j}$

$\operatorname{MinZ}_{2}\left(\operatorname{or} M a x Z_{2}\right)=\sum_{i=1}^{m} \sum_{j=1}^{n} t_{i j} X_{i j}$

$\operatorname{MinZ}_{K}\left(\operatorname{or} \operatorname{Max}_{K}\right)=\sum_{i=1}^{m} \sum_{j=1}^{n} t_{i j} X_{i j}$

$\sum_{j=1}^{n} X_{i j}=1 \quad$ for all $i=1,2, \ldots, m$

$\sum_{i=1}^{n} X_{i j}=1 \quad$ for all $j=1,2, \ldots, n$

$X_{i j}= \begin{cases}1 & \text { عند التخصيص }\end{cases}$

حيث أن: X $=X_{1}, X 2, \ldots . ., X_{n}$ t t $=t_{1}, t_{2}, \ldots ., t_{n}$ = = m = عدد الأشخاص يساوي عدد الوظائف.

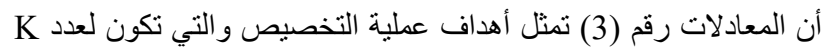

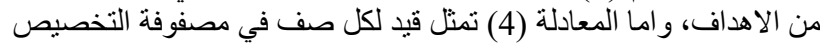

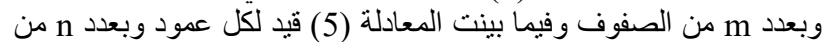
الاعمدة في مصفوفة التخصيص. 
الجدول (2): أولويات أهداف مشكلة التخصيص للانموذج الأول.

\begin{tabular}{|c|c|}
\hline 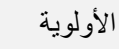 & 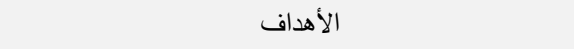 \\
\hline $\mathrm{P}_{1}$ & تحقيق أعلى رغبة للمستجيب بالتخصيص للشعبة المطلوبة. \\
\hline $\mathrm{P}_{2}$ & تحقيق اقل كلفة لعملية التخصيص. \\
\hline
\end{tabular}

الجدول (3): يبين ملخص نتائج عملية التخصيص للنموذج الأول.

\begin{tabular}{|c|c|c|c|c|c|c|}
\hline 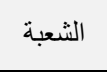 & المستجيب & الثُعبة & المتغير & الر غقدار & الرغية & التخصيص كلفة \\
\hline المالية & 33 & 1 & $\mathrm{X}_{33,1}$ & 5 & أو افق & 48 \\
\hline التدقيق & 7 & 2 & $\mathrm{X}_{7,2}$ & 5 & أو افق & 55.2 \\
\hline والتخطيط & 2 & 3 & $\mathrm{X}_{2,3}$ & 5 & بشدة & 54.3 \\
\hline 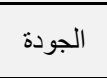 & 1 & 4 & $\mathrm{X}_{1,4}$ & 5 & أشدة & 57 \\
\hline الإداروية & 22 & 5 & $\mathrm{X}_{22,5}$ & 5 & بشدة أو افق & 118.8 \\
\hline شؤون & 5 & 6 & $\mathrm{X}_{5,6}$ & 5 & بشدة & 47.1 \\
\hline و التطوير & 18 & 7 & $\mathrm{X}_{18,7}$ & 5 & أو افق & 76.35 \\
\hline \multicolumn{4}{|c|}{ المجموع حسب النتائج } & 35 & \multicolumn{2}{|c|}{456.75} \\
\hline \multicolumn{4}{|c|}{ قيمة الهدف المطلوب } & 35 & \multicolumn{2}{|c|}{329.7} \\
\hline \multicolumn{4}{|c|}{ قيمة الانحر اف عن الهدف } & $\mathrm{n}_{1}=0$ & \multicolumn{2}{|c|}{$\mathrm{P}_{2}=127.05$} \\
\hline
\end{tabular}

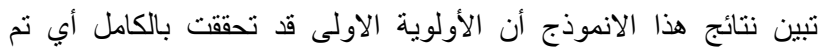

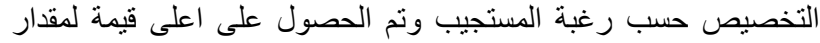
الرغبة ولجميع شعب الكلية وهو او افق بشدة لذا كانت. (n1=0)

أما الأولوية الثانية فقد أنحرفت عن الهدف المحدد بزيادة مقدار اها (127.05)

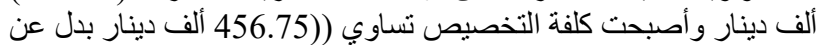

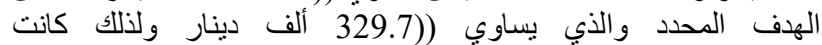
.(P2=127.05)

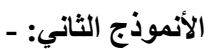

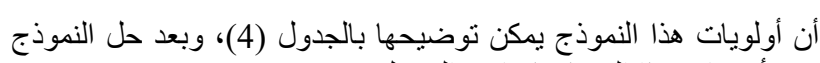
تبين أن نتائج هذا النموذج كما في الجدول توذ (5). الجدول (4): أولويات أهداف مشكلة التخصيص للأنموذج الثاني.

\begin{tabular}{|c|c|}
\hline الأولوية & الأهداف \\
\hline $\mathrm{P}_{1}$ & تحقيق اقل كلفة لعملية التخصيص. \\
\hline $\mathrm{P}_{2}$ & المطلوبة. أعلى رغبة للمستجيب بالتخصيص للشعبة \\
\hline
\end{tabular}

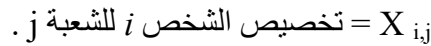

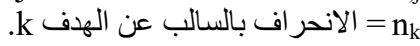
k الانحر اف بالموجب عن الانب عن الهدف =pk

\subsection{2}

أن عدد القيود الهيكلية للمشكلة ب (45) قيد ويمكن التعبير عن أهم القيود الأساسية لنماذج المشكلة بما يأتي:

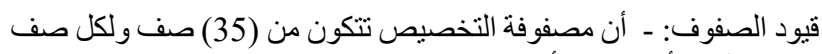
قيد من النوع أصغر من أو يساوي و احد .

قيود الأعمدة: - أن مصفوفة التخصيص تتكون من (7) عمود ولكل عمود قيد من النوع أصغر من أو يساوي و احد.

قيود الأهداف: - أن عملية التخصيص للحالة التطبيقية تتكون من (2) هدف ولكل هدف قيد.

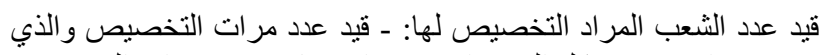

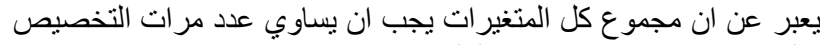

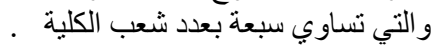
هذا بإضافة شرط عدم السلبية يكون كالاتي:

$$
\text { Xij, di-, di }+\geq 0
$$

إما التعبير الرياضي عن شرط أن تكون متغيرات القرار أعدادا ثنائية يكون كالاتي:

$$
\mathrm{Xij}=0 \text { or } 1
$$

$$
\text { أهداف نموزج التخصيص }
$$

8.2.3

للمشكلة هدفين يمكن توضيحهما كما يأتي:

الهدف الأول: تحقيق اعلى رغبة لاى المستجيب و الذي عبرنا عنه بأتفق بشدة

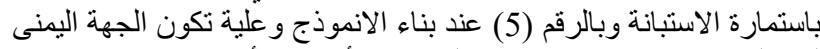

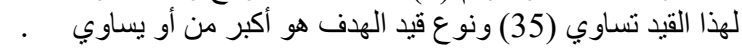

الهدف الثاني: تحقيق اقل كلفة تخصيص، وبما ان أقل كلفة تخصيص هي

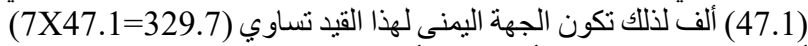

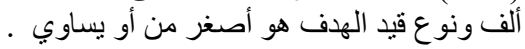

\subsection{4}

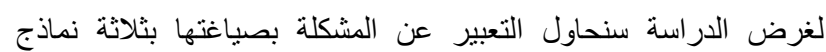

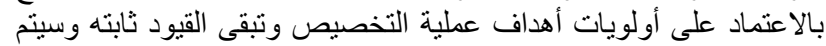

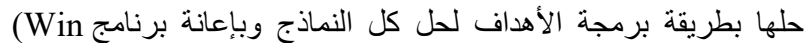
Version (2).

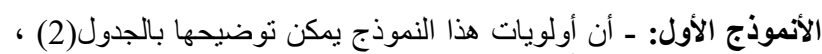

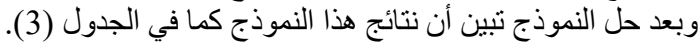


الجدول (5): يبين ملخص نتائج عملية التخصيص للنموذج الثاني.

\begin{tabular}{|c|c|c|c|c|c|c|}
\hline الثعبة & رمز المستجيب & رمز الثعبة & رمز المتغير & كلفة التخصيص & مقدار الرغبة & معيار الرغية \\
\hline المالية & 10 & 1 & $\mathrm{X}_{10,1}$ & 48 & 3 & محايد \\
\hline التدقيق & 12 & 2 & $\mathrm{X}_{12,2}$ & 48 & 3 & محايد \\
\hline والتخطيط & 33 & 3 & $\mathrm{X}_{33,3}$ & 48 & 3 & محايد \\
\hline 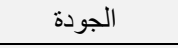 & 35 & 4 & $\mathrm{X}_{35,4}$ & 48 & 4 & أو افق \\
\hline الثؤون الإدارية & 6 & 5 & $\mathrm{X}_{6,5}$ & 47.1 & 4 & أو افق \\
\hline شؤون الطلبة & 5 & 6 & $\mathrm{X}_{5,6}$ & 47.1 & 5 & أو افق بشدة \\
\hline البحث و التطوير & 3 & 7 & $\mathrm{X}_{3,7}$ & 48 & 4 & أو افق \\
\hline \multicolumn{4}{|c|}{ المجموع حسب النتائج } & 334.2 & 26 & \\
\hline \multicolumn{4}{|c|}{ قيمة الهدف المطلوب } & 329.7 & 35 & \\
\hline \multicolumn{4}{|c|}{ قيمة الانحر اف عن الهذف } & $\mathrm{P}_{1}=4.5$ & \multicolumn{2}{|c|}{$\mathrm{n}_{2}=9$} \\
\hline
\end{tabular}

الانموذج أن لهذا النموذج اولوية واحدة وبهدفين، حيث أنحرف الهدف الاول

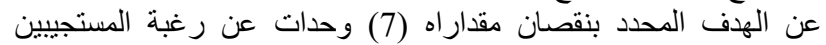

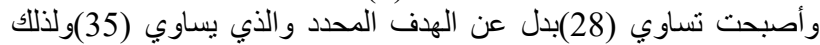

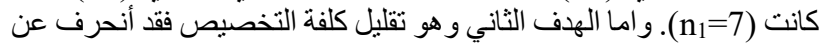

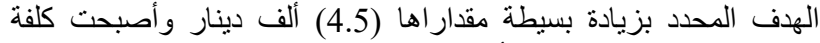

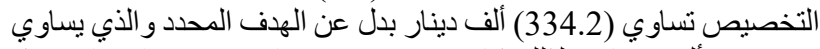

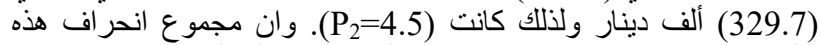
الأولوية وهو مجموع انحر اف الهدف الأول وانت الثاني أب أن:

$$
\mathrm{n}_{1}+\mathrm{P}_{2}=7+4.5=11.5
$$

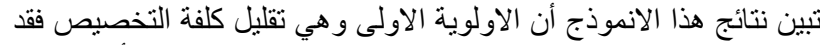

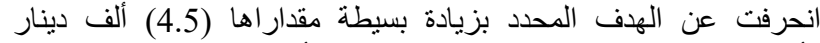

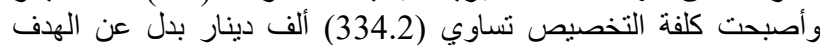
المحدد و الذي يساوي (329.7) ألف دينار ولذلك كانت.(31=4.5)

أما الاولوية الثانية فقد انحرفت عن الهدف المحدد بنقصان مقداراه (9)

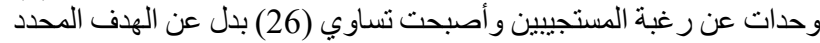
و الذي يساوي (35) ولذلك كانت (n2=9).

الأنموذج الثالث: -أن أولويات هذا النموذج مثساوية بالأهمية وبعد حل

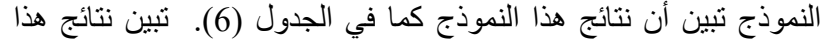

الجدول (6): يبين ملخص نتائج عملية التخصيص للنموذج الثالث.

\begin{tabular}{|c|c|c|c|c|c|c|}
\hline الثعبة & رمز المستجيب & رمز الشعبة & رمز المتغير & 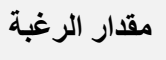 & 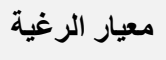 & كلفة التخصيص \\
\hline 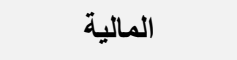 & 33 & 1 & $\mathrm{X}_{33,1}$ & 5 & 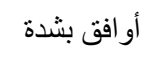 & 48 \\
\hline 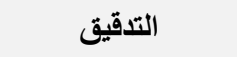 & 12 & 2 & $\mathrm{X}_{12,2}$ & 3 & محايد & 48 \\
\hline الاراسات والتخطيط & 3 & 3 & $\mathrm{X}_{3,3}$ & 3 & محايد & 48 \\
\hline الجودة & 35 & 4 & $\mathrm{X}_{35,4}$ & 4 & أو افق & 48 \\
\hline الثؤون الإدارية & 6 & 5 & $\mathrm{X}_{6,5}$ & 4 & أو افق & 47.1 \\
\hline 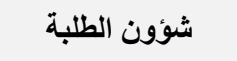 & 5 & 6 & $\mathrm{X}_{5,6}$ & 5 & أو افق بشدة & 47.1 \\
\hline البحث والتطوير & 27 & 7 & $\mathrm{X}_{27,7}$ & 4 & أو افق & 48 \\
\hline \multicolumn{4}{|c|}{ المجموع حسب النتائج } & 28 & \multicolumn{2}{|c|}{334.2} \\
\hline \multicolumn{4}{|c|}{ قيمة الهدف المطلوب } & 35 & \multicolumn{2}{|c|}{329.7} \\
\hline \multicolumn{5}{|c|}{ قيمة الاتحراف عن الهـف } & \multicolumn{2}{|c|}{$\mathrm{n}_{1}+\mathrm{P}_{2}=7+4.5=11.5$} \\
\hline
\end{tabular}


المصادر

[1] ل لايذ، و اثق حياوي " اتخاذ القرارات ذات الدوال الكسرية باستخدام

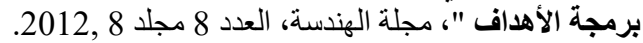

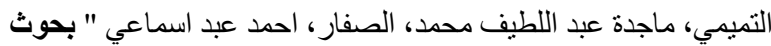

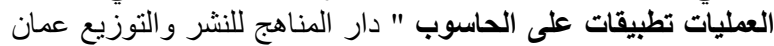

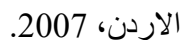

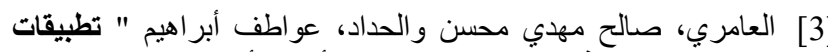
بحوث العمليات في الإدارة "، الطبعة الأولى، أثراء للنشر والته التوزيع،

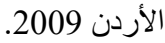

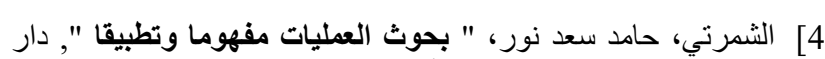
و ائل للنشر في عمان، الطبعة الأولى, 2010.

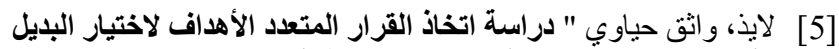

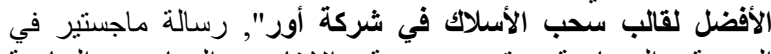

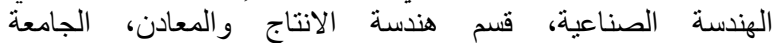

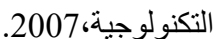

[6] خالد، بوشارب،" دور نموذج البرمجة الخطية متعددة الأهداف في اتخاذ

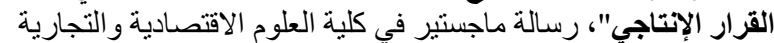

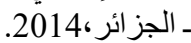

[7] Kepner C. and Tregoe, B."The Rational Manager: A systematic Approach to Problem Solving and Decision Making", McGraw Hill, New York, NY.1995.

[8] Kothari C. R "An Introduction to Operation Research", 3rd edition, Vikas Publishing House PVTLTD, 1992.

[9] Leon, C. and Palacios F Evaluation of Rejected Cases in an Acceptance System with Data Envelopment Analysis and Goal Programming ", Journal of the operational research Society Vol. 6 ,pp. 1411-1420, 2009.

[10] Martel J.M. and Aouni B." Incorporating the DecisionMakers Preferences in the Goal- Programming Model", Journal of the Operational Research Society, Vol. 12, pp.121-132, 1990.
الاستتتاجات والتوصيات

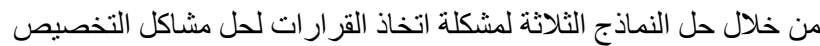
المتعدد المعايير باستخدام طريقة برمجة الأهداف نستنتج ما يأتي: -

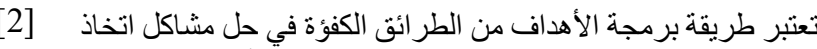

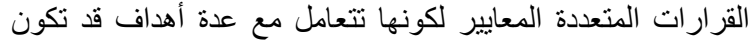
متعارضة أو متناقضة.

النماذج الثلاث المفترضة لعطلية التخصيص أعطت نتائج مختلفة تبعا

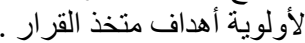

الأنموذج الأول حقق الهدف الأول بدون أب انحر اف (n1=0) و الذي الذي

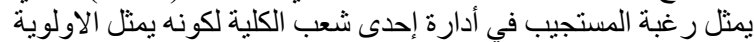

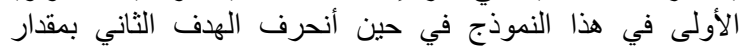

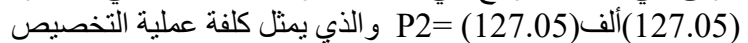
وهي الاولوية الثانية.

الأنموذج الثاني حقق الهدف الأول بانحراف بمقداره (4.5) ألف بأل

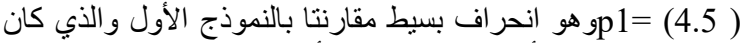

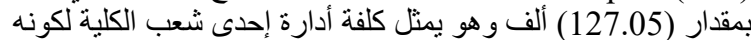

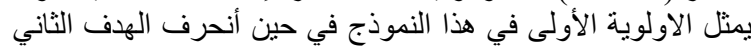

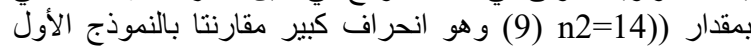
والذي لم ينحرف عن الهذف المحدد.

5. الأنموذج الثالث وفيه تتساوى فيه الأهداف بالأهمية وكان مجموع بهو

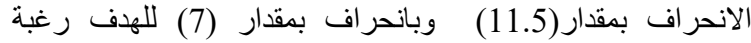
المستجيب و انحر اف بمقدار (4.5) لكلفة التخصيص.

بعد الاطلاع على الاستنتاجات يمكن ان نوصي فيما يأتي:

1 ـتوجيه اهتمام متخذي القرار إلى الاعتماد على أساليب الكمية في عملية اتخاذ قرار اتهم.

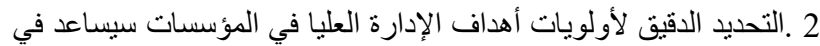
دقة القرار المتخذ، لكون أي تغير في الأولوية ممكن أن يغير الألئ عملية اتخاذ

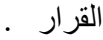

3. اعتماد هذا البحث في عملية اتخاذ القرارات المتعددة المعايير وباستخدام

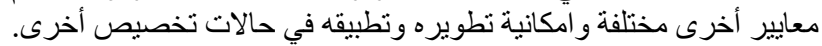




\title{
Linear Goal Programming Method for Optimal Decision Making of Multi-Criteria Assignment Problems
}

\author{
Watheq Hayawi Laith
}

Department of Statistics, College of Administration and Economic, University of Sumer, Iraq

Published online: 30 June 2019

Abstract- The decision making process is the essence of the administrative process and its means of achieving the goals of the service organizations or productivity because the decision contributes mainly to enable them to carry out their administrative activities efficiently and effectively. The assignment process is one of the most important problems affecting the organizations performance because to determine the administrative composition of the organization. The correct decision making to the assignment problem and the right person in the right place will increase the efficiency and effectiveness of the organization. The previous methods to solve these problems depend on a single criterion such as cost, profit, time and so on. In this paper, three models were formulated for the of multi-criteria assignment problem. The first model multiple objective for present assignment problem when the assignment matrix is square, while the second and third models are a non-square assignment problem. The second model is the number of rows is greater than the number of columns while the third model where the number of columns larger than the rows. The second model is applied to the assignment problem of unit managers in the college of Administration and Economics based on two criteria: deferring the employee's desire to leader of task and the other criterion of assignment cost and using goal programming method. The data were collected by questionnaire.

Keywords- N/A. 